\title{
HOW CAN 3D GIS BE USED TO BETTER STORE, INTEGRATE AND COMMUNICATE RESULTS OF ENVIRONMENTAL IMPACT ASSESSMENTS?
}

\author{
Monika A. Swiderska ${ }^{1}$, Claire Ellul ${ }^{1}$ \\ ${ }^{1}$ Department of Civil, Environmental and Geomatic Engineering, University College London, Gower Street, London, WC1E 6BT - \\ (monika.swiderska.16, c.ellul)@ucl.ac.uk
}

KEY WORDS: 3D GIS, GIS, environmental datasets, Environmental Impact Assessment, 3D mapping, data integration, 3D georeferencing

\begin{abstract}
:
In the United Kingdom, the process of conducting the Environmental Impact Assessment (EIA) is a legal requirement for projects of considerable size or those that can affect the environment. EIA results are presented as an Environmental Statement, which includes multiple long reports often covering thousands of pages on large developments and includes complex information that is hard to understand for the intended audience of planners and the general public. To facilitate understanding, environmental professionals often use 2D maps. However, this approach is only partially useful, as it is difficult to locate specific information or to identify information related to a specific location on a very large site. This paper presents a pilot study into whether 3D GIS provide an alternative to better present, integrate and communicate the EIA results, with specific focus on three core environmental datasets: noise, air quality and bats' flight paths, in relation to the building model of a real world development site. We focus in particular on off-the-shelf technologies that would be available to a standard Environmental Consultancy not having access to bespoke development, and will be usable by the planners, public and other decision makers, and look at tasks including 3D georeferencing, integration and visualisation of the data to be explored. It was concluded that while 3D GIS has a potential for environmental modelling, current technology and standards of data collection limit the practical use of 3D GIS at this stage.
\end{abstract}

\section{INTRODUCTION}

\subsection{Background}

In the United Kingdom, the process of conducting the Environmental Impact Assessment (EIA), as part of planning application, is a legal requirement for projects of considerable size or those that can affect the environment. The requirements for the EIA are governed by a number of regulations including the 'Town and Country Planning (Environmental Impact Assessment) Regulations 2017' (DCLG, 2017).

\subsection{Current Challenges}

Typical factors under consideration in an EIA include issues such as noise, air quality, traffic, protection of wildlife and so forth, with details of the current situation, the projected situation following the development process, and any mitigation put in place to reduce the project's impact on the environment (further details in Section 2.1). However, current planning regulations state that EIA results are to be presented in a written report named the Environmental Statement (ES).

The report comprises over 1200 pages of text, maps and tables stored in three folders. In general, the number of factors included in the EIA process is dependent on the type of a development and on the decision of local authorities as to which environmental factors should be considered. Furthermore, there are no rules in place that regulate the length of the ES. Thus, the reports are often long and the data included not easily accessible due to the inherent complexity of the document and to the fact that it is presented in printed format. Typically, the printed or digital form of the ES would be available to interested parties and it the best source of information about the EIA process.

Many of the factors considered as part of the EIA process have a geospatial component, and the importance of these factors is often related to spatial distributions of their effects (Antunes et al. 2001). Indeed, given the spatial (location) nature of many of the factors to be considered in an EIA, off-the-shelf Geographical Information Systems are commonly used by EIA consultants as part of the preparation of the ES. However, currently environmental data is commonly processed and presented in 2D GIS (Eta et al., 2014), with the main output being paper maps for inclusion in the report. This means that no access is provided to the data used to compile the maps, and the potential of interacting with both the data and the resulting analysis outputs - e.g. via an online web mapping tool - is lost.

Additionally, many of the projects for which an EIA is required are large developments, that are modelled in 3D via options such as Building Information Modelling (Level 2 BIM is required on Government Projects in the UK since 2016, Cabinet Office 2011). Static 2D paper maps do not allow the intrinsically $3 \mathrm{D}$ nature of such projects to be explored.

\subsection{Exploring the Potential of 3D GIS}

A recent report for the Scottish Government, entitled "Use of Digital and 3D Technology in Planning for New Development" noted the potential of 3D visualisation to help to communicate the Environmental Impact better to planners and the general public (Miller et al., 2016). Furthermore, given its ability to geo-reference data both horizontally and vertically, 3D GIS has the potential to 'index' the data presented in the report, allowing users to click on a component of a 3D model and access relevant information (e.g. as a PDF).

This paper presents a pilot study into whether environmental consultants can use commercially available $3 \mathrm{D}$ GIS tools ${ }^{1}$ to

1 Note that we focus on commercially available off the shelf tools as these are currently used within such consultancies. Additionally it is unlikely that EIA experts have access to developers to produce and - importantly - maintain bespoke software. 
communicate the results of the EIA in a more accessible way to a wider audience, including: environmental professionals, spatial planners, governmental organisations and the general public. Rather than exploring the problem from a GIS experts perspective, we explore the problem taking into account both the level of GIS skills (and in particular 3D GIS skills) of an environmental consultant producing such a report - i.e. someone who uses GIS in the course of their work, but is primarily an environmental expert.

We explore two aspects of the problem. Firstly, can the data currently used in EIA be georeferenced as required - which datasets included in the ES are fit-for-purpose in terms of visualisation, integration and georeferencing using 3D GIS and what, if anything, needs to be done when preparing these datasets (i.e. during the EIA creation process) to ensure that they can be used as part of an interactive presentation. Secondly, what tools are available to present the information in a usable manner. to assess which visualisation tool can provide the best results for 3D GIS EIA.

EIA and spatial planning are typically carried out by specialists. Thus, this project was undertaken in partnership with Quod a recognised spatial planning and environmental consultancy. This enabled us to make use of data from a pre-approved EIA created for a real construction project in London, although the specific location of the data has been shifted for the purposes of publication. The reason to choose an already approved project was that yet to be approved projects are very much commercially sensitive.

The paper is structured as follows: we first present a summary of the Environmental Impact Assessment process in the UK, including the types of spatial data that form part of this process. Current use of GIS in EIA is briefly reviewed, along with the potential benefits provided by 3D GIS (both for visualisation and for more in depth exploration of semantic information). The data used in our tests is then presented, followed by a review of available $3 \mathrm{D}$ software to identify tools most appropriate for visualisation. Methods used to configure the data appropriate for the software are then described, and results presented. The paper concludes with a discussion as to whether current tools and data do support wider use of 3D GIS in EIA as proposed, highlighting challenges still to be addressed.

\section{LITERATURE REVIEW}

\subsection{Environmental Impact Assessment}

The purpose of an EIA is to provide a mechanism to protect the environment from potentially harmful developments (DCLG, 2017). In principle, an EIA should consider all positive and negative effects of the proposed development to help the local authority make an informed decision whether the development should be granted (ibid.). Moreover, an EIA allows measures to be taken at the design stage of the proposal to avoid, reduce or mitigate unacceptable or unfavourable environmental effects.

In the United Kingdom, the legislation is covered by a range of regulations, the most common being the 'Town and Country Planning (Environmental Impact Assessment) Regulations 2017' (DCLG, 2017). Not all development projects require an EIA, but those that do include: large infrastructure developments such as airports, roads, nuclear power stations, crude oil refineries, quarries, industrial and agricultural developments and others as described by the sets of EIA Regulations within the UK (ibid.).

An example of components to be included in the resulting report include (DCLG 2017) 2 :

(a) a description of the proposed development comprising information on the site, design, size and other relevant features

(b) a description of the likely significant effects of the proposed development on the environment

(c) a description of any features of the proposed development, or measures envisaged in order to avoid, prevent or reduce and, if possible, offset likely significant adverse effects on the environment

(d) a description of the reasonable alternatives studied by the developer, which are relevant to the proposed development and its specific characteristics, and an indication of the main reasons for the option chosen, taking into account the effects of the development on the environment

Typically, the factors included in the EIA would fall into one of these categories: land use, land price, population density, socioeconomic levels, road accessibility, railway accessibility, air quality, ground water quality, noise level, biological content, historical value, archaeological and visual importance (Eta et al., 2014). However, depending on the site assessed, the factors included in the EIA may vary. It is also important to note that the ES generated by the EIA is legally required to be created as a report (DCLG, 2017).

2.1.1 Current Use of GIS in EIA: The use of GIS seems to be a natural choice to help presenting the complex outcomes of the EIA. Indeed, Davis and Haklay (2004) reported that GIS was applied to the EIA process from the early 1970s, but it was not until 1990s that GIS began to be used in the EIA mapping on a regular basis. 2D maps are currently considered to be an integral part of the ES (Eta et al., 2014), but the use of 3D mapping in the context of the EIA is still being researched (Danese et al., 2008, Lai et al., 2010).

\subsection{D GIS in EIA}

3D GIS offer two potential avenues to assist with the management of EIA outputs - firstly, interactive visualization, and secondly the 'Information System' aspect, which allows users to drill down into the semantics and additional information associated with an object, and potentially to undertake some analysis on the data presented.

2D GIS is recognised a first-choice tool in EIA mapping (Appleton and Lovett, 2005, Atkinson and Canter, 2011). As currently utilised in an EIA, 2D maps are static. Both 2D and 3D GIS do, however, offer the option of a more dynamic, interactive view on the EIA, as highlighted by Miller et al. (2016) who note the benefit of 3D visualisations within the planning process. These would allow users to explore the plans, outcomes and mitigation factors.

Looking beyond EIA specifically, advancing technology and growing popularity of visualisations, provides an indication of the potential of this approach. It is recognised that $2 \mathrm{D}$ maps can be inadequate for displaying complex geographic phenomena and in some cases having a 3D representation of a dataset unlocks new ways of analysis (Pouliot et al., 2016). A full copy of the legislation can be found here: http://www.legislation.gov.uk/uksi/2017/571/pdfs/uksi_20170571_en.pdf, Accessed $12^{\text {th }}$ May 2018 
3D visualisations represent objects with width, depth and height, and can provide visual images (Miller et al., 2016) of a site and information. As such, they can be constructed using data embedded in local coordinate systems (as is frequently the case in BIM). 3D GIS additionally, require a spatial reference (ibid.), which allows the integration of various datasets as the spatial component provides a link between them - permitting the EIA to include both project specific data but also data about the wider context of the project (neighbouring buildings, traffic counts, open spaces, transport infrastructure), and any other available environmental data for the location. That, in turn, gives 3D GIS the capacity to capture and present spatial data in more useable way, allowing both visual and semantic information to be explored. Gristina et al. (2016) highlight the potential of this approach in the context of road cadastres.

To date, it would appear that 3D GIS is not widely used in EIA, and the authors were not able to identify literature to provide an evidence of the research done specifically on 3D GIS in EIA.

\section{DATA}

\subsection{Development Site}

The development site modelled in this project is located in London, UK. The choice of the site was made by Quod, who stated that it is a typical construction project requiring an EIA. The proposal states that the building is 22 storeys in its tallest part, dropping down to 6 and 8 storeys towards the north end. The ES for this site had already been completed, and provided the source data for our investigation. Due to sensitivity of the data and number of parties involved in the process of creating the EIA, in this particular case, the use of the ES was the only option as the raw datasets were not made available.

\subsection{Assessing EIA Output for 3D GIS}

Given the different factors to be explored, the potential number of datasets under consideration is significant and an initial list of 32 datasets was identified with in the ES. To focus the work, Quod indicated that some datasets have a higher business importance than others, and these were selected for this initial project. It is also felt that they provide a good representation of the wider datasets that could be used in future. Of the suggested datasets, further consideration was given as to how the data is included in the EIA process, in what form it is included and whether it can be visualised in 2D GIS (including $\mathrm{x}$ and $\mathrm{y}$ coordinates) and/or 3D (2D and height or $\mathrm{z}$ coordinate).

From the available 32 datasets, 3 were chosen: noise, air quality and bats' flight paths.

3.2.1 Noise: Noise data was available in the ES in a printed table that was then digitised ${ }^{3}$. The dataset consisted of 4 columns with $\mathrm{dB}$ numbers and information about the floor (storey) and façade the data was modelled for.

\footnotetext{
3 It is anticipated that in future this and other data sources will be made available directly in digital format, during the creation of the ES. This was not the case here as we were only able to access a pre-existing ES due to commercial sensitivity of such statements.
}

3.2.2 Air Quality: Air quality modelling is a complex process as the actual values of $\mathrm{NO}_{\mathrm{x}}$ in the atmosphere depend on several factors including weather, wind and time of day (Fenger et al., 1998). The data included in the ES was annual mean concentration values for a centre of a $1 \mathrm{~km}^{2}$ grid cell in the chosen location, shown in Table 1.

\begin{tabular}{|l|l|l|l|}
\hline X Coordinate & Y Coordinate & NOx & $\mathrm{NO}_{2}$ \\
\hline 522500 & 182500 & 54.5 & 33.1 \\
\hline
\end{tabular}

Table 1 Annual mean concentration values in $\mu \mathrm{g}$ m-3 for a $1 \mathrm{~km} 2$ cell grid. Data was acquired from Defra as per the ES.

As the values are annual means for the larger area, the actual data had to be modelled in more detail before being included in the 3D GIS. The approach used was developed by TuckettJones and Reade (2017).

3.2.3 Ecology (Bats): The information about the occurrence of bats was provided as a report. The dataset included the date and time of a survey taken and the species of bats observed. However, it did not include the exact locations of the bat observations. Thus, a database of flight paths was mocked up based on the information provided.

\section{SELECTING VISUALISATION SOFTWARE}

As key component of this research was the selection of the most appropriate visualisation platform, with selection criteria being guided by 'Company A'. These are presented in Table 2 .

\begin{tabular}{|l|}
\hline 3D Extension \\
\hline Ease of Use for non-GIS specialists \\
\hline Installation of specialist software required \\
\hline Availability of instructions/courses/trainings \\
\hline Reads different data formats \\
\hline License (free vs paid) \\
\hline Options to style the data \\
\hline Ability to switch layers on and off \\
\hline Integration with future online platform \\
\hline Type of output \\
\hline Quality of output \\
\hline Cost (including training of staff, additional license etc.) \\
\hline Time to prepare the model \\
\hline
\end{tabular}

Table 2 List of requirements for Visualisation Software

Following a preliminary evaluation, three options were considered in more detail ${ }^{4,5}$ : ArcScene, Google Earth and 3D PDF, with the former considered as Quod already hold the software in house, and the latter two offering free options for $3 \mathrm{D}$ visualisation and hence the potential to distribute the EIA results widely. As currently there is no capability within the partner organisation to develop and maintain a more sophisticated software or code-based solution, only off-the-shelf products were considered.

${ }^{4}$ The study was conducted in the summer of 2017 and thus, currently there might more viable products available on the market.

${ }^{5}$ CityGMLwas disregarded despite permitting modelling in a 3D environment as it is not specifically designed for environmental data that would be generated by an EIA. 


\section{METHODS}

\subsection{Converting AutoCAD data into GIS formats}

The model of a proposed development was provided by Quod in the AutoCAD format (DWG). As AutoCAD files are mainly used by architects and developers, the model was very detailed and consisted of 281,147 elements. This made the processing extremely challenging. AutoCAD models are not easily read by GIS software. Differences exist between the AutoCAD format and GIS as these systems are "conceptually misaligned" (Boyes et al., 2015). As well as conversion, the model also required georeferencing as it was provided in the local coordinate system of the project.

The first approach tested was to import the model into Autodesk Revit, the software designed to analyse Building Information Models. In this case, BIM proved to be insufficient as the data was not collected and created in mind to be used as BIM. Another attempt to georeference the model was made using ArcMap and the AutoCAD model translated into Esri shapefile using FME Workbench 2017. A third attempt to georeference the model was made using an Offsetter transformer in FME. Additionally, an attempt was made to translate the AutoCAD model into KML file that could be used in the final Google Earth models.

\subsection{Modelling the Datasets}

The modelling of the environmental data was done using pgAdmin III and PostGIS. Additionally, to overcome conversion issues, a simplified model of the development was created using SQL that included each of the designed buildings. The coordinates of the modelled building were obtained from the map in ArcMap. The 3D noise and air quality models were created in SQL and stored as vertical polygons. Bats' flight paths were created using a similar approach with the difference, that in this case, class multilinestring was used instead of polygon. The models were then styled in ArcScene.

\subsection{Background Mapping}

The Ordnance Survey (OS) MasterMap was downloaded from Edina Digimap and styled using in ArcScene to distinguish different features of the base map. Additionally, the building heights shapefile was obtained from Edina Digimap and uploaded into ArcScene project.

\subsection{Exporting Environmental Models to KML}

Three possible options were explored to export the model to a format readable by Google Earth. The formats considered were KML and KMZ. The process involved trial and error methods

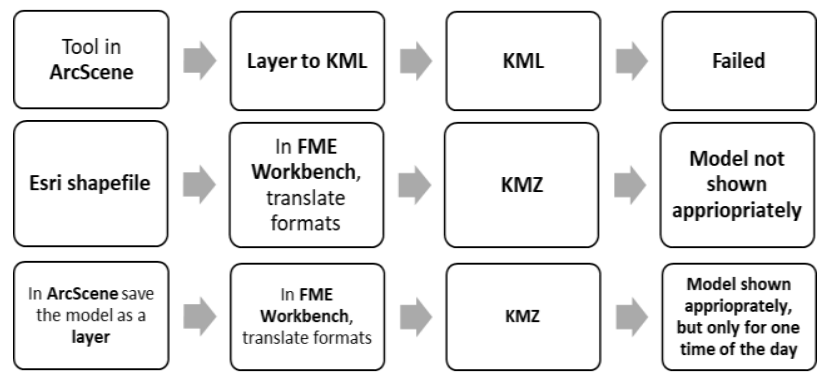

Figure 1 The workflows of creating Google Earth readable file and some of the attempts failed. Details of each workflow are presented in the Figure 1.

\subsection{Exporting the Model to 3D PDF}

Several attempts were made to export the project to 3D PDF. The first approach attempted was to translate Esri shapefile to 3D PDF using FME. The model was deemed unsuitable as the styling of the data was not preserved. The next attempt was to save the styled model as a layer in ArcScene and then translate it to 3D PDF using FME. This approach also proved inappropriate as only one layer could be translated at the time, and PDF cannot be edited to add more layers to the final document. Furthermore, an attempt was made to translate a group layer. This approach failed as well as FME was not able to create the workspace. The attempt to convert a KML file into a 3D PDF using FME was also unsuccessful as PDF file was empty. The final attempt of exporting the model into a 3D PDF file was made using ArcScene and FME. Firstly, in ArcScene the project was exported as a VRML file using built-in tools. Then, the VRML was translated into 3D PDF in FME.

\section{RESULTS}

\subsection{ArcScene}

The converted AutoCAD model, Building Heights and the base map were added to each project to give context to the visualisation. Note, however, that AutoCAD model that was included in ArcScene project could not be explored, as the software froze. Additionally, the final output is missing the polylines that enabled the comparison between floors of the building.

6.1.1 The Noise Model: The noise model presented in Figure 2 has a difference in displayed colours. This indicates the scale of noise as it changes throughout the models. The green coloured façades are those of low $\mathrm{dB}$ recordings and are located mostly in the eastern and northern sides of the building, while the red indicates high noise levels. The green-red colour scale was chosen by Quod.

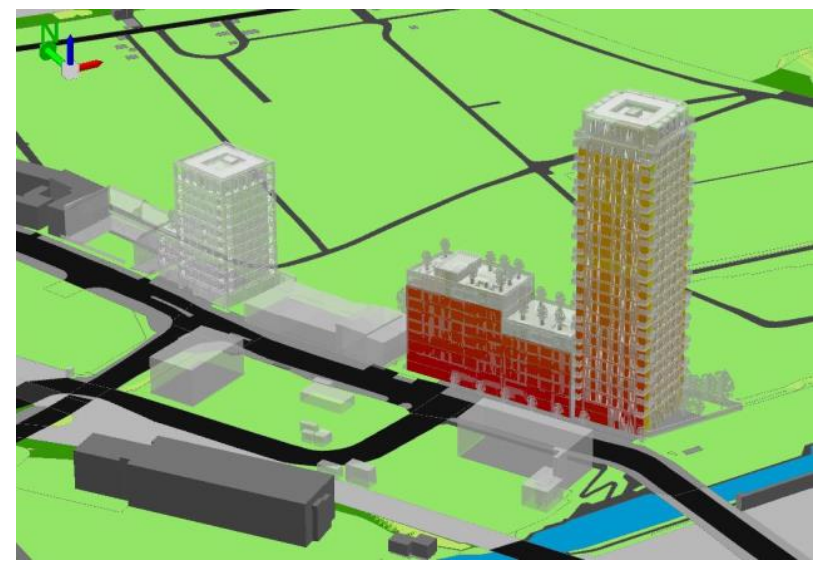

Figure 2 The zoomed in view of the western and southern sides

6.1.2 The Air Quality Model: The air quality model shown in Figure 3 has the scale colour of navy blue through light blue to green with the darkest blue representing the poorest air quality and green representing lower levels of $\mathrm{NO}_{\mathrm{x}}$.

However, in both models it was found that while ArcScene 
offered generally useful visualisation options, the width of the outlines of each individual vertical polygon cannot be adjusted even though the software offered such an option.

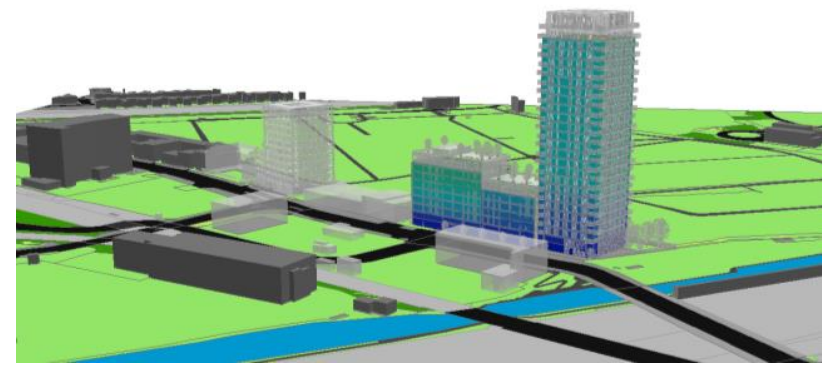

Figure 3 The air quality model viewed from the west side of the development.

6.1.3 Bat's Flight Paths Model: The model of bats' flight paths is shown on Figure 4. Bats were noticed on the east side of the building. Thus, the paths were mapped accordingly and displayed as brown lines.

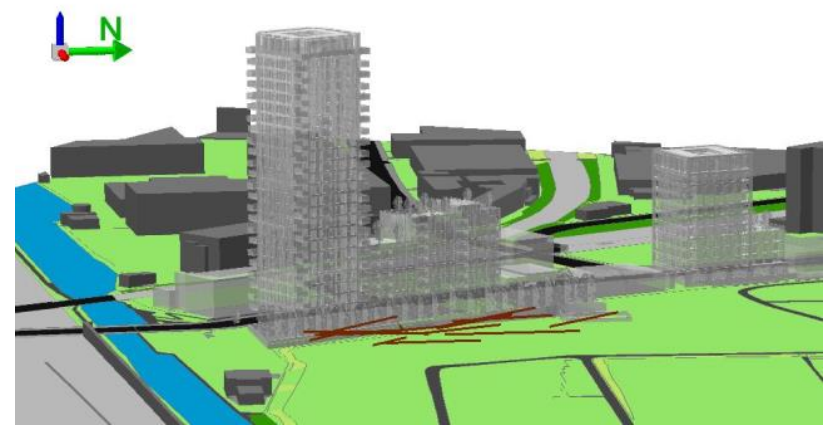

Figure 4 The zoomed in bats' flight paths

\subsection{Google Earth}

The colours used to style the data on the Google Earth models are consistent with those used in ArcScene. The visualisations include a simplified model of the building as the complex AutoCAD model could not be translated into KML.

6.2.1 The Noise Model: The noise model is presented in Figure 5 shows the model in the morning hours, while Figure 6 in the afternoon. There is a difference between them due to received sunlight. This could affect the interpretation of the results as the colours are shown in different shades. Furthermore, the vertical polygons are semi-transparent allowing to see through, which was not the intention during the design stage.

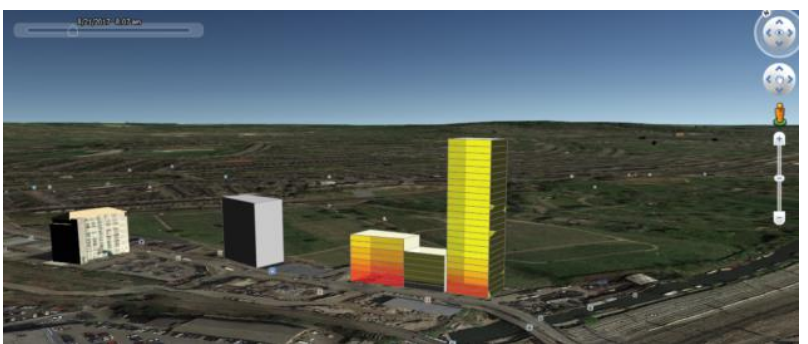

Figure 5 The noise model at 8.00 am as seen from the southeast side of the development

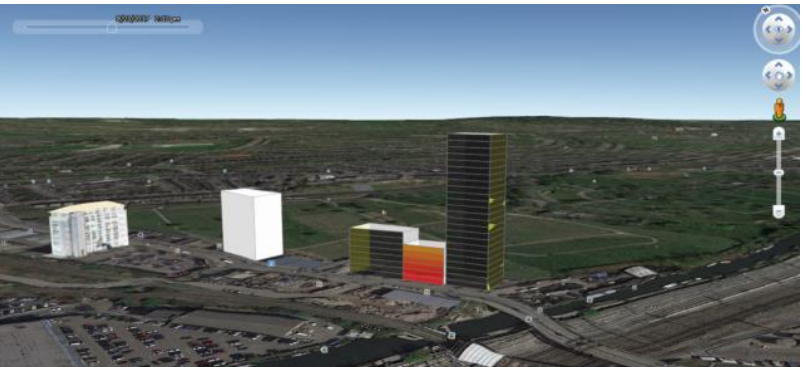

Figure 6 The noise model at $2 \mathrm{pm}$ as seen from the south-east side of the development.

6.2.2 The Air Quality Model: The air quality model is presented in Figure 7. The model also suffers from issues with the difference in colour presentations, which makes the data interpretation difficult.

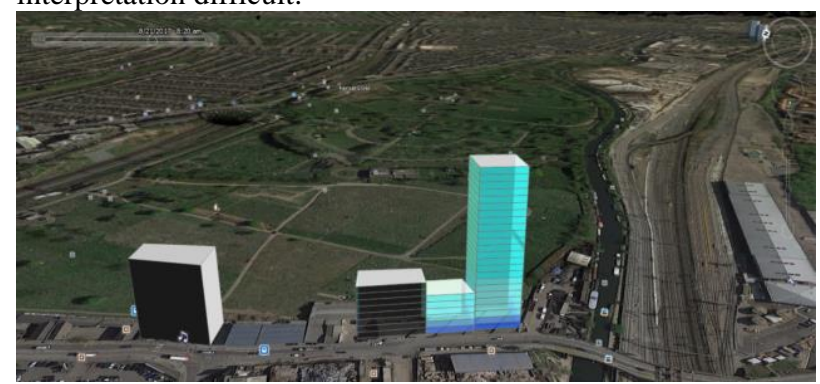

Figure 7 The air quality model at $8.20 \mathrm{am}$ as seen from the south-east side of the development.

6.2.3 The Bats' Flight Paths Model: The bats' flight paths model is shown on Figure 8. The paths are coloured in brown as this was the colour used in the ArcScene model. However, the lack of contrast highlights the potential problem of presenting the same model in different software. Additionally, the simplified model of the building is included.

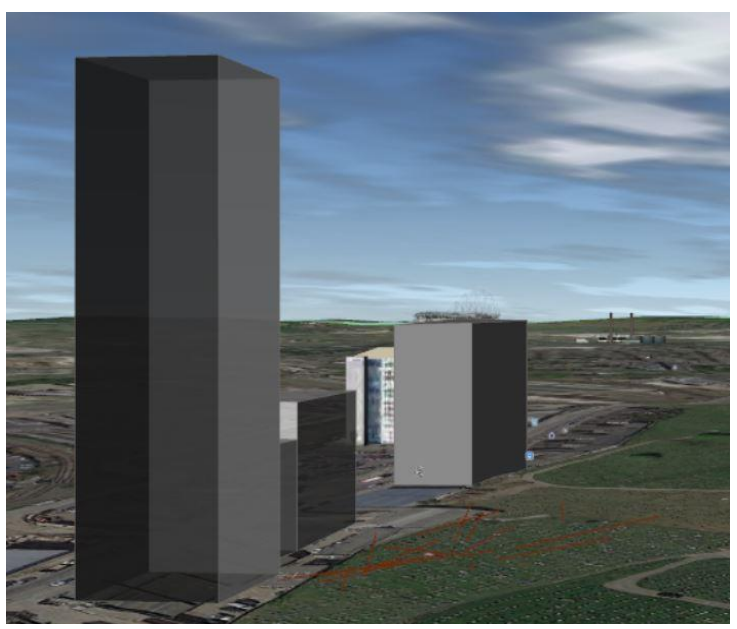

Figure 8 The zoomed in model of bats' flight paths. The model includes simplified buildings.

\subsection{D PDF}

As with the previous case, the AutoCAD model could not be exported to 3D PDF, and a simplified model was used. 
6.3.1 The Noise Model: As shown in Figure 9 the colours used to style the model are consistent with those used in ArcScene and Google Earth to allow easier interpretation of the results. The simplified model of the building is semi-transparent to allow noise styling to be displayed.

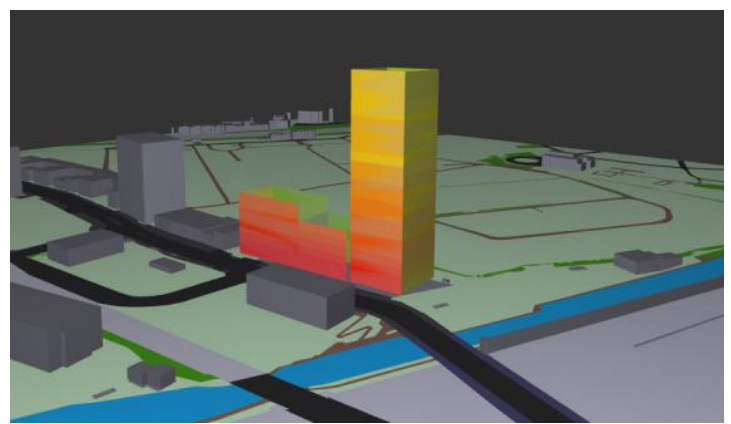

Figure 9 The noise model with the semi-transparent simplified building.

6.3.2 The Air Quality Model: The air quality model is presented in Figure 10 and follows the styling chosen for previous models.

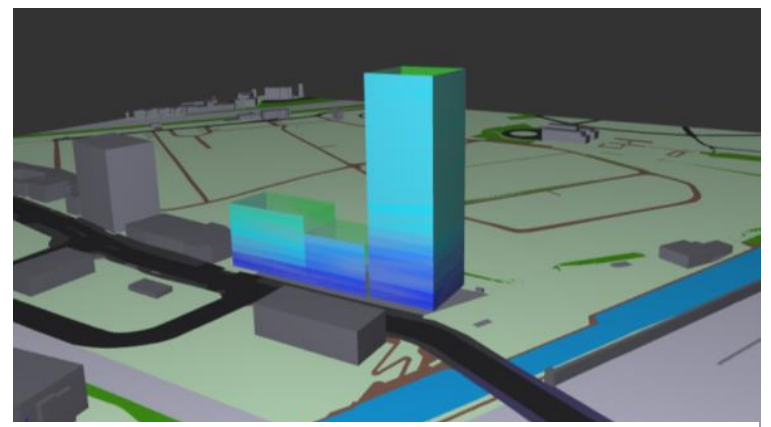

Figure 10 The air quality model with the semi-transparent simplified building model.

6.3.3 The Bats' Flight Paths Model: The dataset was not included in the project exported from ArcScene in a VRML file, indicating an issue with the ArcScene software when exporting linear 3D data. All figures showing the models are missing legends as none of the software used provided this functionality.

\subsection{Software and Data Interoperability Summary}

The processes described above highlighted a number of challenges with the different data formats and software, which are summarised in Table 3:

\begin{tabular}{|c|c|c|c|}
\hline & ArcScene & 3D PDF & Google Earth \\
\hline $\begin{array}{l}\text { CAD } \\
\text { model }\end{array}$ & $\begin{array}{l}\text { CAD data } \\
\text { conversion } \\
\text { possible via } \\
\text { FME. } \\
\text { Geolocation } \\
\text { and conversion } \\
\text { via Revit not } \\
\text { possible. }\end{array}$ & $\begin{array}{l}\text { CAD data } \\
\text { could not be } \\
\text { used. } \\
\text { Simplified } \\
\text { version } \\
\text { required }\end{array}$ & $\begin{array}{l}\text { CAD data could } \\
\text { not be used, } \\
\text { simplified } \\
\text { version } \\
\text { required. }\end{array}$ \\
\hline $\begin{array}{l}\text { Noise } \\
\text { Data }\end{array}$ & $\begin{array}{l}\text { Visualisation } \\
\text { successful }\end{array}$ & $\begin{array}{l}\text { Conversion } \\
\text { successful via } \\
\text { ArcScene to } \\
\text { VRML and } \\
\text { then to FME }\end{array}$ & $\begin{array}{l}\text { Conversion } \\
\text { successful via } \\
\text { ArcScene Layer } \\
\text { and FME }\end{array}$ \\
\hline Air & Visualisation & Conversion & Conversion \\
\hline
\end{tabular}

\begin{tabular}{|l|l|l|l|}
\hline $\begin{array}{l}\text { Qualit } \\
\text { Data }\end{array}$ & successful & $\begin{array}{l}\text { successful via } \\
\text { ArcScene to } \\
\text { VRML and } \\
\text { then to FME }\end{array}$ & $\begin{array}{l}\text { successful via } \\
\text { ArcScene Layer } \\
\text { and FME }\end{array}$ \\
\hline $\begin{array}{l}\text { Bats } \\
\text { Data }\end{array}$ & $\begin{array}{l}\text { Visualisation } \\
\text { successful }\end{array}$ & $\begin{array}{l}\text { No } \\
\text { conversion } \\
\text { possible. }\end{array}$ & $\begin{array}{l}\text { Conversion } \\
\text { successful via } \\
\text { ArcScene Layer } \\
\text { and FME }\end{array}$ \\
\hline
\end{tabular}

Table 3 Software/Data Interoperability

\section{DISCUSSION}

This paper presented a pilot study into whether environmental consultants can use commercially available 3D GIS tools to communicate the results of the EIA in a more accessible way to a wider audience, focussing on the suitability of current EIA data for inclusion in a 3D model, and on the potential of 3D GIS as a data visualisation and integration/exploration tool.

To a certain extent, the research has shown the potential of this approach, with four EIA datasets integrated into one visualisation, within three software packages, two of which (Google Earth and 3D PDF) offer potential for wider distribution of the EIA results to non-experts. Given the spatial nature of the data involved in an EIA, it can be anticipated that further datasets could also be incorporated as necessary.

Once in these environments, it was possible to explore the data through visualisation, interact the data and find out additional semantic information and, potentially, to link through to additional data such as reports (e.g. by including links as 3D points with the URL of the required section of the report).

However, the issues encountered highlight the fact that an extensive amount of technical pre-preparation work, and indeed changes to how an EIA is created, is required before the potential of 3D GIS can become a reality. Limitations within the 3D GIS tools explored were also encountered. Both these aspects are discussed in further detail here:

\subsection{Requirements for EIA Data Preparation}

7.1.1 Environmental Datasets: Most of the environmental datasets in the ES are presented as text. Even though many of them could potentially be presented in $3 \mathrm{D}$, the data collection does not consider this at the moment. Another problem is that each dataset is different and must be considered separately when it comes to 3D modelling. Thus, the approach used to model one e dataset may not be suitable for the other due to the nature of the data and it is not possible to develop one method that would fit all.

Issues encountered include:

- $\quad$ Positional accuracy of the dataset - the heights given for the various noise calculations did not match the floor heights within the CAD model

- $\quad$ Currency of the dataset - the air quality dataset in the ES did not correspond, in terms of the number of storeys, to the digital model of the building

- $\quad$ Flight path details for the bats were not captured in a suitable spatial format and had to be inferred. In practice, such information is captured using, as indicated in the ES, the observations through noise receivers. These could be geolocated into the $3 \mathrm{D}$ model and expert advice obtained as to how to infer the flight paths from the readings. 
As well as highlighting issues, these findings also further reenforce the need for a digital version of the ES rather than a paper version. This would allow data quality to be assessed across multiple datasets, and for the data to be updated more easily as the inevitable changes in a project's design occur.

\subsection{Interoperability issues with 3D GIS}

7.2.1 CAD and 3D GIS: Four methods were explored on how to georeference the AutoCAD model and convert it into GIS formats. An attempt was also made to convert the AutoCAD model into 3D PDF. The only conversion that was successful was using FME translating AutoCAD to shapefile. Although each software package seems to have the capacity to handle different formats, this project has proven that in practice, with a complex model, this may not be the case. The problems encountered using Revit, show that the AutoCAD, BIM and GIS integration is not complete and there is a need to improve the interoperability between software packages.

More specifically, the level of detail of the AutoCAD model prevented the smooth translation for the GIS modelling and it makes the analysis, translations and error handling more difficult, sometimes causing software to freeze. To avoid this issue, there is a need to explore an automated 'generalisation' process that would preserve the main features of the model while reducing it to a level suitable for inclusion in a GIS. Equally, GIS software vendors may need to explore options to ensure that their software handles complex geometry.

To address the georeferencing issue, surveyors should measure the exact coordinates of the proposed building and submit them as part of the planning process. The information could be then incorporated by the architects and designers into the Revit or AutoCAD model.

7.2.2 3D GIS to 3D GIS: While many of the conversions from ArcScene to 3D PDF or Google Earth were successful, some loss of data (the bats' flight paths) did occur, highlighting a need to further investigate interoperability not only across CAD and GIS but within GIS itself. The tools used - in particular FME from Safesoft - could perhaps be considered state-of-the-art in terms of commercial-off-the-shelf interoperability functionality, but even within this context problems were encountered.

Of particular note is the route to Google Earth, which involved the creation of a VRML output. This means that any associated semantic information is potentially lost.

\subsection{Visualising and Exploring the Data}

Once interoperability issues were overcome, all three of the tools examined offered the potential to visualise and explore the resulting $3 \mathrm{D}$ datasets.

7.3.1 ArcScene: Of the three options, ArcScene was the most powerful in terms of the volume of data that could be handled, and also in terms of the functionality offered to interactively style the 3D data. It was also the only tool that could visualise the complex CAD model. As a certain level of expertise is required to drive GIS tools, this option could be used by an EIA consultancy when preparing a report for dissemination, provided issues such as the problem with visualising vertical polygons (e.g. the noise data) can be overcome. Indeed, Quod are currently ArcGIS users. Currently,
ArcScene cannot be linked to the online platform. However, the development of ArcGIS Online platform by Esri may lead to the creation of a $3 \mathrm{D}$ platform in the future.

7.3.2 Google Earth: The Google Earth platform is free to all users and allows one to switch the layers on and off. It can also be embedded in a website and provides inbuilt hyper-linking functionality to allow users to click on a 'marker' on the map and line to an online document. Time-bar driven navigation may also be relevant to allow users to explore how a site will change over time, for example changes to shadows. While Google Earth platforms do not allow the editing of data this may not be a disadvantage for non-expert users. However, the visual appearance of models changed depending on the time of day, leading to potential mis-interpretation of results.

7.3.3 3D PDF: 3D PDF is not a GIS, but a visualisation format as it does not store spatial data. However, it has the capacity to present $3 \mathrm{D}$ models. It can be easily opened using a free application provided by Adobe Acrobat Reader, a format with which many users are familiar. There are, however, problems associated with this format. As the application has no means of editing or styling the data, and once a 3D PDF is saved, it cannot be changed, this again would be more relevant as a tool for non-expert users. However, both ArcScene and FME are needed to create 3D PDF files, requiring additional expertise and software expense. Finally, 3D PDF models can only be uploaded online as attachments and could not underpin an interactive website showcasing the ES.

7.3.4 Selecting a Visualisation Option: Despite the advantages of Google Earth and 3D PDF, after discussion with Quod, it was decided that neither of these tools are in fact suitable for presenting EIA results. In particular, Google Earth could not be used due to the problems with data styling and shadows are too significant to be ignored and the expertise and software required to create 3D PDF was not present in house. Furthermore, missing legends can be considered a problem. Neither of the 3D software used allow to add a legend to 3D views. Adding a legend manually using picture editing software could would produce legends with colours not fully matching ones used in the software.

\section{CONCLUSIONS AND FUTURE WORK}

The aim of this paper was to provide a preliminary exploration of the potential use of 3D GIS as a presentation tool for an Environmental Statement, using off-the-shelf tools which fall within the level of GIS expertise within an Environmental Consultancy. The results highlighted the potential of this approach, and do provide clear suggestions as to how EIA data management tasks can be modified in order to enable such visualizations at a later date.

However, the above assessment highlights that, currently, none of the 3D GIS solutions investigated provided a good match for the needs of EIA reporting and the GIS skills level - unlike 2D GIS, 3D GIS is not yet mature enough to be easily deployed by non-experts. Although it could be assumed that current off-theshelf technology should allow the integration of the datasets and transfer between different formats, the interoperability between software still limited. ArcScene, Google Earth and 3D PDF met some of the requirements set out in the beginning, but none of these met all of them. However, as ArcScene was required to make Google Earth and 3D PDF models, it should be noted that this software was a crucial element of this project. 
Additionally, data handling is limited by hardware processing power and graphics performance. Therefore, a powerful computer is needed to handle the robust datasets and complex translation processes. This contrasts with a requirement for a low-cost easy to use approach for results dissemination.

The complexities of the process highlight the level of difficulty, and the GIS skills required may not be within reach of an EI consultant used to creating 2D maps and Quod have concluded that they will not explore this option further at the moment.

\subsection{Future Work - Improving Data Collection Methods}

This preliminary research has shown that there is a wide range of environmental datasets that have a potential be used in 3D modelling, yet in many cases the information is not documented in a way that would allow the data to be re-used. Therefore, environmental consultants need structured standards for data collection that take account of 3D GIS. This would allow the better integration of datasets for modelling.

Furthermore, considering the problems encountered during the AutoCAD model translation to different formats, the solutions for the AutoCAD model inclusion should be explored. Typically, AutoCAD models are created by developers, architects or engineers who do not consider 3D GIS requirements, with a high level of detail due to engineering requirements. One solution could be to produce a simplified version of a model for GIS use, also ensuring that this is georeferenced. It is a common practice in the industry to survey the chosen site before the development starts and would be relatively easy to include the exact coordinates of a proposed building on a 2D map. Libraries such as GDAL may also offer useful tools to address this challenge (although a simple conversion does not overcome the 'too much geometry' issue).

Increasingly, web-based solutions offer potential in this regard, with open software such as Cesium $\mathrm{JS}^{6}$ becoming increasingly prominent as a tool for web-based 3D GIS functionality. Such an approach- coupled with bespoke software development would allow the visualisation to be integrated with other tools to allow a more targeted exploration of the EIA results, with future potential for scenario modelling. QGIS 3.0 - which was released following this study - provides 3D visualisation functionality that should also be explored.

\section{ACKNOWLEDGEMENTS}

The authors would like to thank Alistair Walker and Environmental Planning team from Quod. Without their support and initiative this project would not be possible.

\section{REFERENCES}

Antunes, P Santos, R and Jardáo, L (2001) The application of Geographical Information Systems to determine environmental impact significance, Environmental Impact Assessment Review 21 (2001) $511-535$.

Appleton, KJ and Lovett, AA (2005) GIS-based visualisation of development proposals: reactions from planning and related professionals, Computers Environment and Urban Systems, 29 (2005), pp. 321-339
Atkinson, S.F. and Canter, L.W. (2011) Assessing the cumulative effects of projects using geographic information systems. Environ. Impact Assess. Rev., 31, 457-464.

Boyes, G Thomson, C and Ellul, C (2015) Integrating BIM and GIS: Exploring the use of IFC space objects and boundaries, GISRUK 2015, 15 April 2015 - 17 April 2015

Cabinet Office (2011), Government Construction Strategy', Available from: https://www. gov.uk/government/uploads/system/uploads/attachment_ data/file/61152/Government-Construction-Strategy_0._Pdf [Accessed 12 ${ }^{\text {th }}$ May 2018].

Danese, M Las Casas, G Murgante, B (2008) 3D Simulations in Environmental Impact Assessment. In: Gervasi O., Murgante B., Laganà A., Taniar D., Mun Y., Gavrilova M.L. (eds) Computational Science and Its Applications - ICCSA 2008. ICCSA 2008. Lecture Notes in Computer Science, vol 5072. Springer, Berlin, Heidelberg

Davis, M Haklay, M (2004) 3D GIS: Adding Value to the Environmental Impact Assessment. GEO:connexionUK (March 20) pp. 30-31

Eta, JB Adepoju, MO Halilu, SA, Mohammed, SO Adeluyi, A (2014) Geographic Information Systems (GIS) As an Indispensable Tool for Environmental Impact Assessment (EIA), IOSR Journal of Environmental Science, Toxicology and Food Technology, Vol 8, 32-39. DOI 10.9790/2402-081223239

Fenger, J Hertel, O Palmgren, F (1998) Urban Air Pollution European Aspects. Springer.

Gristina, S., Ellul C, and Scianna A. (2016) "Developing A 3D Road Cadastral System: Comparing Legal Requirements And User Needs." ISPRS Annals of the Photogrammetry, Remote Sensing and Spatial Information Sciences 4: 223

Lai, PC Kwong, KH Mak, ASH (2010) Assessing the applicability and effectiveness of $3 \mathrm{D}$ visualisation in environmental impact assessment, Environment and Planning B: Planning and Design, Vol 37, 221-233. DOI: $10.1068 / \mathrm{b} 34141$

Loh, E Dawood, N N and Dean, JT (2007) Integration of 3D tool with environmental impact assessment (3D EIA), ASCAAD 2007 Conference, Alexandria, Egypt, November 2830 in, Em'body'ing virtual architecture. ASCAAD

Miller, D Morrice, J McKeen, M Donaldson-Selby, G Wang, C Munoz-Rojas, J (2016) Use of Digital and 3D Technology in Planning for New Development. Report for Scottish Government

Pouliot, J Hubert, F Wang, C Ellul, C Rajabifard, A (2016) 3D Cadastre Visualization: Recent Progress and Future Directions, 5th International FIG 3D Cadastre Workshop, 18-20 October 2016, Athens, Greece

The Department of Communities and Local Government (2017) Guidance. Environmental Impact Assessment

Tuckett-Jones, B and Reade, T (2017) City Air Quality at Height - Lessons for Developers \& Planners, WSP- Parsons Brinckerhoff

\footnotetext{
${ }^{6}$ https://cesiumjs.org, Accessed $12^{\text {th }}$ May 2018
} 